\title{
Dynamical Crystallites of Active Chiral Particles
}

\author{
Zhi-Feng Huang, ${ }^{1}$ Andreas M. Menzel, ${ }^{2,3}$ and Hartmut Löwen $\oplus^{2}$ \\ ${ }^{1}$ Department of Physics and Astronomy, Wayne State University, Detroit, Michigan 48201, USA \\ ${ }^{2}$ Institut für Theoretische Physik II, Heinrich-Heine-Universität Düsseldorf, Universitätsstraße 1, D-40225 Düsseldorf, Germany \\ ${ }^{3}$ Institut für Physik, Otto-von-Guericke-Universität Magdeburg, Universitätsplatz, 2, 39106 Magdeburg, Germany
}

(Received 27 April 2020; accepted 2 October 2020; published 17 November 2020)

\begin{abstract}
One of the intrinsic characteristics of far-from-equilibrium systems is the nonrelaxational nature of the system dynamics, which leads to novel properties that cannot be understood and described by conventional pathways based on thermodynamic potentials. Of particular interest are the formation and evolution of ordered patterns composed of active particles that exhibit collective behavior. Here we examine such a type of nonpotential active system, focusing on effects of coupling and competition between chiral particle selfpropulsion and self-spinning. It leads to the transition between three bulk dynamical regimes dominated by collective translative motion, spinning-induced structural arrest, and dynamical frustration. In addition, a persistently dynamical state of self-rotating crystallites is identified as a result of a localized-delocalized transition induced by the crystal-melt interface. The mechanism for the breaking of localized bulk states can also be utilized to achieve self-shearing or self-flow of active crystalline layers.
\end{abstract}

DOI: 10.1103/PhysRevLett.125.218002

Systems of self-propelled or self-spinning active particles are intrinsically out of equilibrium. Operating with self-sustaining energetic sources or propulsive forces, the corresponding active dynamic processes should no longer be governed by the traditional relaxational pathways directed by the minimization principle of thermodynamic potentials as in near-equilibrium samples of passive particles. Instead, the system evolution is controlled by nonpotential, nonrelaxational dynamics, a category that has been known to exhibit a variety of complex states, such as evolving ordered and defected patterns [1-3], spatiotemporal chaos with persistent dynamics [4,5], or glassy behavior [6] as found in physical and biochemical pattern-forming systems like fluid convection, liquid crystals, chemical reactions, and many biological processes [1]. Complex dynamical behavior has also been observed in active colloidal materials both experimentally and computationally, varying from phase separation [7-12], dynamical clustering [7,13-16], and active glass [17,18] to traveling [19-22] or rotating [23-25] crystals.

Among these dynamical phenomena of active matter, a common feature is the collective motion of the constituent particles or building blocks in homogeneous [24-29] or phase-separated [7-12] liquid and/or gas states and ordered phases with different crystalline symmetry [19-25,30-34].

Published by the American Physical Society under the terms of the Creative Commons Attribution 4.0 International license. Further distribution of this work must maintain attribution to the author(s) and the published article's title, journal citation, and DOI.
In many cases, the self-propulsion of active particles, coupled with interparticle interactions, is the driving factor underlying various forms of collective behavior [7,11-13,16-22]. Recent experimental and theoretical studies also showed that the self-spinning or self-circling alone (of active spinners or rotors [35-40]) can generate spatiotemporal collective states among the interacting chiral particles [8-10,23-33,41-48]. A typical example is the edge current flow of rotors generated at rigid boundary walls; this edge mode induces the collective, unidirectional flow of neighboring rotors, which either decays into the confined sample interior of a liquid or gas phase [24-29] or causes the rotation of the circular sample in a crystalline or jammed phase [24,25].

Although much effort has been devoted to investigating either of these two mechanisms of self-propulsion and selfspinning, effects of their mutual coupling are much less explored. Also less understood is the corresponding crystallization process, for which a statistical continuum description that can access large length and timescales much beyond the restrictions encountered in discrete particle-based approaches, has still been lacking. Here, by introducing a continuum density-field description that is nonpotential and nonvariational, we show that the coupling and competition between self-propulsion and self-spinning result in a surprisingly rich behavior of nonrelaxational dynamical crystallized states. They feature both translational and rotational collective motion, governed by persistent dynamics. Two types of transition for active crystalline patterns are identified, i.e., bulk travelinglocalization and interfacial localized-delocalized transitions, each mediated by a crossover regime showing 
dynamical frustration of active chiral particles. Of particular interest is the effect of the controlled crystal-melt interfaces, leading to an emergent state of self-rotating crystallites embedded in a homogeneous active melt, or self-shearing or wriggling flow of crystalline layers.

The active system here is described by a local particle density variation field $\psi$ and a local polar particle orientation field $\mathbf{P}$, the dynamics of which are governed by

$$
\begin{gathered}
\partial_{t} \psi=\nabla^{2}\left[\epsilon \psi+\left(\nabla^{2}+q_{0}^{2}\right)^{2} \psi-g \psi^{2}+\psi^{3}\right]-v_{0} \nabla \cdot \mathbf{P}, \\
\partial_{t} \mathbf{P}=\left(\nabla^{2}-D_{r}\right)\left(C_{1} \mathbf{P}+C_{4}|\mathbf{P}|{ }^{2} \mathbf{P}\right)-v_{0} \nabla \psi+\mathbf{M} \times \mathbf{P},
\end{gathered}
$$

where $\epsilon$, together with the average density $\psi_{0}$, controls the transition between homogeneous (liquid) and crystalline phases, $v_{0}$ is the self-propulsion strength, $D_{r}$ is the rotational diffusion constant, and $\mathbf{M}=M \hat{z}$ represents the strength of self-spinning caused by an active torque. When $\mathbf{M}=0$ we recover the previous active phase field crystal (PFC) description $[19,20]$.

Here we focus on $C_{1}>0$, counteracting any spontaneous polarization by, e.g., rotational diffusion, to preclude any explicit alignment interactions and be consistent with related experiments. Our description can be derived from a microscopic particle-based formulation and dynamical density functional theory (DDFT) (see Supplemental Material [49]). All the model parameters are rescaled and dimensionless, giving a diffusion timescale and a spatial scale set by the periodicity of the ordered phase with $q_{0}=1$.

It is noteworthy that Eqs. (1) and (2) could be reduced to a gradient, relaxational form only when $v_{0}=M=0$ for passive particles, giving $\partial_{t} \psi=\nabla^{2} \delta \mathcal{F} / \delta \psi$ and $\partial_{t} \mathbf{P}=$ $\left(\nabla^{2}-D_{r}\right) \delta \mathcal{F} / \delta \mathbf{P} \quad$ with $\mathcal{F}=\int d \mathbf{r}\left\{\psi\left[\epsilon+\left(\nabla^{2}+q_{0}^{2}\right)^{2}\right]\right.$ $\left.\psi / 2-g \psi^{3} / 3+\psi^{4} / 4+C_{1}|\mathbf{P}|^{2} / 2+C_{4}|\mathbf{P}|^{4} / 4\right\}$, a combination of the PFC free energy [84-87] and a Landau expansion of the polarization field. Thus, for any active system with nonzero $v_{0}$, the corresponding system dynamics is nonrelaxational, i.e., does not follow the minimization of $\mathcal{F}$. We examine this nonpotential system through a series of numerical simulations. Each starts either from a homogeneous state with random initial conditions or from initially small crystalline nuclei, with system sizes ranging from $128 \times 128$ to $2048 \times 2048$ grid points (around 230 to 60000 density peaks) subjected to periodic boundary conditions. The system parameters are chosen as $\left(\epsilon, g, D_{r}, C_{1}, C_{4}\right)=(-0.98,0,0.5,0.2,0)$, while values of $M, v_{0}$, and $\psi_{0}$ are varied to control the competition between self-propulsion and self-spinning.

Our simulations indicate that, in the bulk state of active crystals, three characteristic regimes of system dynamics can be identified. As shown in Fig. 1(a), the propelling-spinning competition leads to a sharp transition between a unidirectionally traveling ordered state driven by particle selfpropulsion at large enough $v_{0}$ and small $M$ [Fig. 1(b)] and a localized or arrested crystalline state with vanishing

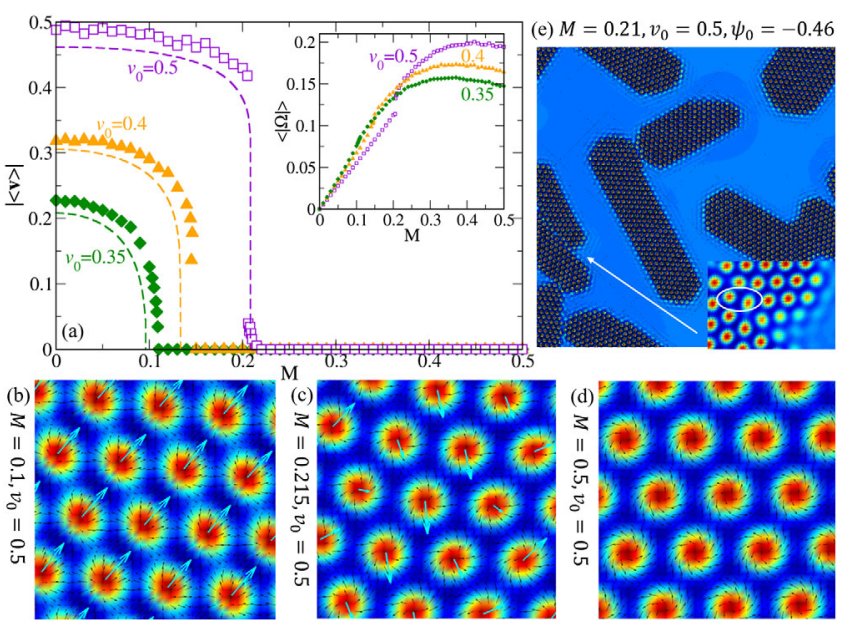

FIG. 1. (a) Magnitude of average density-peak velocity $|\langle\mathbf{v}\rangle|$ as a function of $M$, for $\psi_{0}=-0.3$ and $v_{0}=0.35,0.4$, and 0.5. Dashed curves represent analytic results of Eq. (5) assuming $\hat{q}_{0} \simeq q_{0}=1$. Inset: $\langle|\Omega|\rangle$ measuring particle self-spinning. (b)-(d) Snapshots of parts of the $\psi$ profiles in three characteristic regimes, with the density peaks shown in red, their velocities $\mathbf{v}$ indicated by large arrows, and the polarization field $\mathbf{P}$ by fine arrows. (e) Snapshot of migrating crystallites in an active melt (see Supplemental Material, Video S2 [49]). Inset: Enlarged portion of a two-grain impinged corner, with a penta-hepta dislocation.

velocity $\mathbf{v}$ of each density peak at large enough $M$ [Fig. 1(d)]. The effect of active torque causes the selfspinning or localized self-circling of individual chiral particles and hence the localization of each density peak, which is consistent with previous DDFT results for noncrystallized states [88]. It thus induces the arrest of the whole pattern as observed here. This traveling-localization transition can be accompanied by phase transformations between ordered structures as induced by particle self-spinning when $M$ increases. Examples include transformations from a traveling rhombic or distorted hexagonal structure to a localized hexagonal phase [Figs. 1(b)-1(d)], or from traveling square to traveling rhombic to localize hexagonal structures at large active drive $v_{0}$ (Fig. 2).

In a narrow crossover regime near the transition threshold $\left(M \sim M_{c}\right)$, the incompatibility between two dynamical effects dominated by self-propelled translation and spinning-induced localization becomes explicit. When these two competing dynamics are of similar degree, none of the

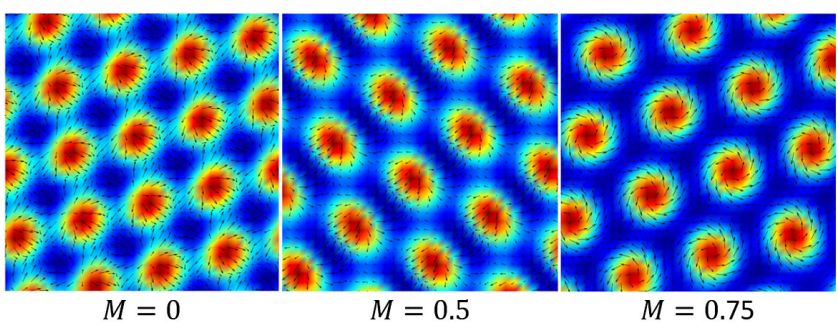

FIG. 2. Self-spinning-induced phase transformation of crystalline patterns with increasing $M$ (at $v_{0}=1, \psi_{0}=-0.4$ ). 
corresponding optimal collective behaviors can be achieved, leading to the local dynamic frustration of active particles [see Fig. 1(c)] or a wavy, swinging translative motion of the whole crystalline pattern characterized by alternative regions of density peaks traveling at varying directions (see Supplemental Material, Video S1 [49]). The balancing of translation and localization also leads to a phenomenon of migrating crystallites. As shown in Fig. 1(e) and Video S2, during the evolution and coarsening of faceted crystallites or grains, the density peaks are localized within each grain, while the whole crystallites travel within the coexisting medium of homogeneous melt, impinging and coalescing with each other.

The above results can be further understood by rewriting Eq. (2) in terms of a local divergence field $S=\nabla \cdot \mathbf{P}$ and the self-spinning field $\Omega=(\nabla \times \mathbf{P})_{z} / 2$; for $C_{4}=0$,

$$
\begin{gathered}
\partial_{t} S=C_{1}\left(\nabla^{2}-D_{r}\right) S-2 M \Omega-v_{0} \nabla^{2} \psi, \\
\partial_{t} \Omega=C_{1}\left(\nabla^{2}-D_{r}\right) \Omega+\frac{1}{2} M S .
\end{gathered}
$$

Equation (4) indicates that $S$ serves as an effective source for nonzero $\Omega$ in the steady state, generating particle selfspinning locally, as confirmed numerically in the inset of Fig. 1(a). Nonzero $S$ also drives the propagation of density patterns when entering Eq. (1), while its own source is, in turn, provided by the variation of the density field [see $v_{0} \nabla^{2} \psi$ in Eq. (3)]. Simultaneously, the term $M \Omega$ in Eq. (3) causes the damping of $S$ and plays the role of an inhibitor that hinders the particle migration, leading to the effect of localization observed in the simulations.

Given the linear form of Eqs. (3) and (4), it is straightforward to express the Fourier components of $S$ and $\Omega$ in terms of those of density $\psi$ in the nonequilibrium steady state with a constant pattern migration speed $v_{m}$. For the hexagonal phase, in one-mode approximation

$$
\begin{aligned}
v_{m}^{2}= & \frac{2}{3 \hat{q}_{0}^{2}}\left\{\hat{q}_{0}^{2} v_{0}^{2}-2\left[C_{1}^{2}\left(\hat{q}_{0}^{2}+D_{r}\right)^{2}-M^{2}\right]\right. \\
& \left.+\sqrt{\hat{q}_{0}^{4} v_{0}^{4}-16 M^{2} C_{1}^{2}\left(\hat{q}_{0}^{2}+D_{r}\right)^{2}}\right\},
\end{aligned}
$$

if $v_{m}^{2}>0$ and $|M| \leq \hat{q}_{0}^{2} v_{0}^{2} /\left[4 C_{1}\left(\hat{q}_{0}^{2}+D_{r}\right)\right]$; otherwise $v_{m}=0$. This analytic result indicates that there exists a critical threshold $M_{c}$ (or $v_{0 c}$ ); when $|M|>M_{c}$ (or $\left.v_{0}<v_{0 c}\right)$ the active crystal is localized with $v_{m}=0$. $\hat{q}_{0}$ is the selected wave number of the ordered pattern, difficult to be determined analytically for a nonpotential system [1-3]. Our simulations indicate that $\hat{q}_{0}$ is in the vicinity of $q_{0}$, an approximation used in evaluating Eq. (5) as presented in Fig. 1(a) without any parameter fitting. We find a reasonably good agreement between analytic and numerical results for $v_{m}$, with the deviations attributed to the employed one-mode approximation.
Remarkably, the effect of chiral particle self-spinning, which leads to the bulk structure localization identified above, can be utilized to generate a further localized-delocalized transition in the interfacial state and, consequently, a phenomenon of crystallite self-rotation or self-shearing. The crystal-melt interface is set up through a sufficiently steep gradient of the "temperature"-type parameters $\epsilon$ and $C_{1}$ (corresponding to the spatial variation of, e.g., chemicals or heat sources controlling homogeneous vs crystalline phases in experiments). Our obtained results are robust against specific realizations of the setup, as verified in simulations. Below, we present results for a kink-type two-phase profile

$$
\epsilon=\frac{1}{2}\left\{\left(\epsilon^{\text {in }}+\epsilon^{\text {out }}\right)+\left(\epsilon^{\text {out }}-\epsilon^{\text {in }}\right) \tanh \left[\left(r-r_{0}\right) / \Delta\right]\right\},
$$

with the same form for $C_{1}$. This represents a circular cavity of radius $r_{0}$, enclosing a crystallite embedded in an outside coexisting homogeneous medium of active melt. Similar kinktype profiles can be set up for other interfacial geometries. Here we set $\left(\epsilon^{\text {in }}, \epsilon^{\text {out }}, C_{1}^{\text {in }}, C_{1}^{\text {out }}\right)=(-0.98,0,0.2,1)$ and $\Delta$ around $1-5$ grid spacings.

Simulation results for a circular cavity are presented in Fig. 3, showing three regimes of crystallite self-motion: (i) self-translation dominated, (ii) self-rotation dominated, and (iii) the transition between them, as a result of the competition between self-propelled translative particle motion, interface-induced tangential motion of density peaks, and localization through particle self-spinning. In regime (i) for small $M$, high-density blocks constantly crystallize from the active melt at one side of the cavity, propagate across it, and remelt into the homogeneous medium at the other side, as seen in Fig. 3(b) and Video S3 [49]. At the same time, the whole crystallite still rotates slowly, with small but nonzero averaged angular velocity $\langle|\omega|\rangle$ [estimated as the orientation change rate of $\langle\mathbf{v}\rangle$, given the dominance of translative motion, with the center of rotation located outside the crystallite; see Fig. 3(a)]. The maximum magnitude of the average translational velocity $|\langle\mathbf{v}\rangle|$ (among realizations of different $\psi_{0}$ ) decreases with increasing $M$, due to stronger localization through particle self-spinning. At large enough $M$ [regime (ii)], persistent self-rotation of the faceted crystallite about the cavity center is observed, as illustrated in Fig. 3(c) and Video S4 [49]. The direction of crystallite rotation (clockwise) is opposite to that of individual particle self-spinning (counterclockwise). The maximum averaged rotation rate $\langle|\omega|\rangle$ (here $|\omega|=|\mathbf{v}-\langle\mathbf{v}\rangle| / r$ ) is reduced at larger $M$ with enhanced bulk localization.

This phenomenon of self-rotating crystallites can be understood by examining the spatial variation of the polarization field $\mathbf{P}$. At the cavity boundary, the average polarization of a boundary density peak at the melt side is weaker than at the inner crystalline side, generating a local spatial gradient of $\mathbf{P}$ (and $\Omega$ ) that is suppressed asymmetrically by its vicinity to the interface. Thus, the active drive at the inner 


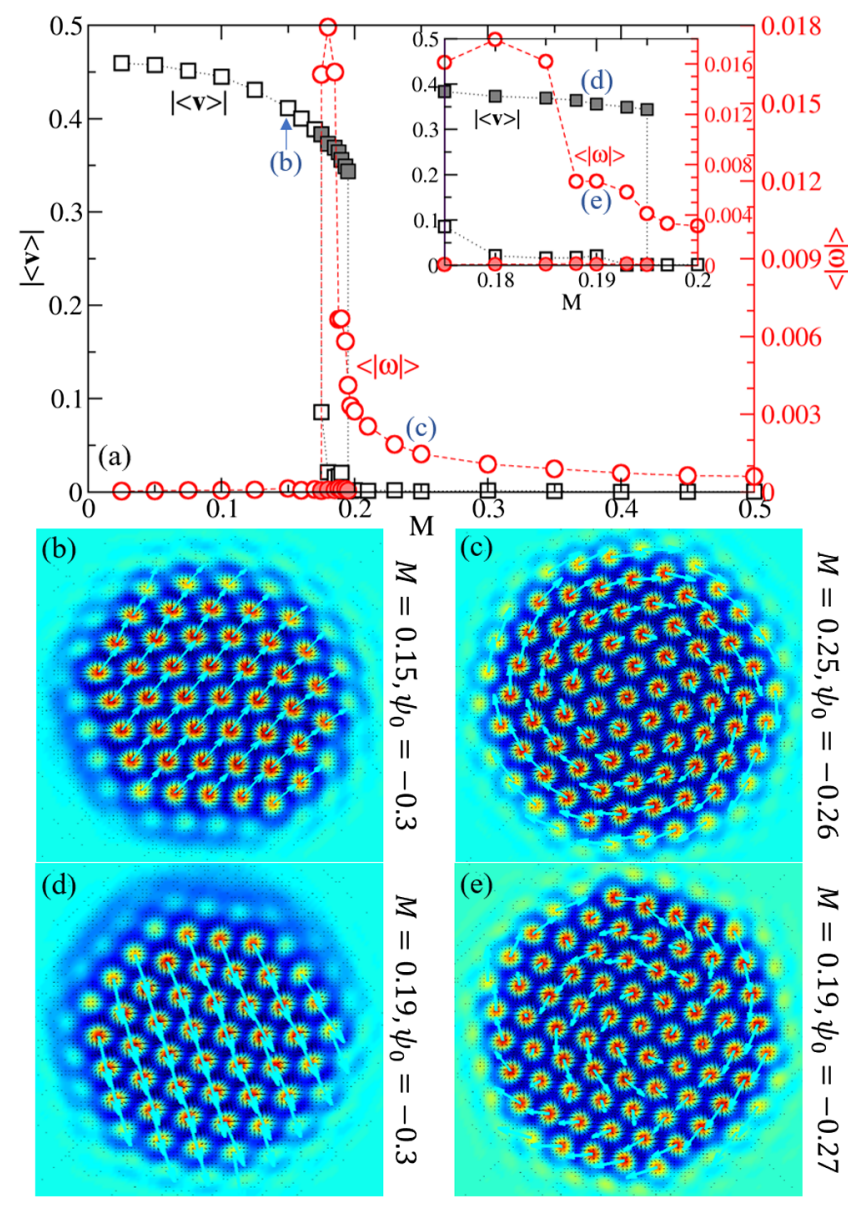

FIG. 3. (a) Averaged translational and rotational velocities $\left(|\langle\mathbf{v}\rangle|\right.$ and $\langle|\omega|\rangle$ at $\left.v_{0}=0.5\right)$ as a function of $M$, containing translation- and rotation-dominated regimes for crystallites in a circular cavity and a transitional regime (shaded symbols) enlarged in the inset. (b)-(e) Snapshots of crystallite patterns simulated [as marked in (a)], with $\mathbf{v}$ vectors indicated.

side of each boundary peak dominates over that of its outer side, causing the corresponding self-flow of the interfacial crystalline layer. It subsequently overcomes the localization of (i.e., delocalizes) interior particles via collective dynamics and drives the overall self-rotation of the crystallite. The direction of self-rotation follows the orientation of the net polarization at the inner side of interfacial peaks and thus, interestingly, is opposite to that determined by the chirality of individual self-spinning. When the sign of $M$ is reversed, both directions of self-spinning and self-rotation are reversed. The mechanism here is different from that underlying the rotation or edge flows of active spinners found in previous studies of no-flux rigid boundary walls; there the chirality of the boundary or edge flow is the same as that of the individual spinners or rotors [24-29].

In the transitional regime, the crystallite shows doubledegenerate behaviors, one dominated by self-translation and the other by self-rotation, as depicted in Figs. 3(d) and 3(e), respectively. In the latter, although the overall crystallite self-rotates as driven by the interfacial layer, some inner

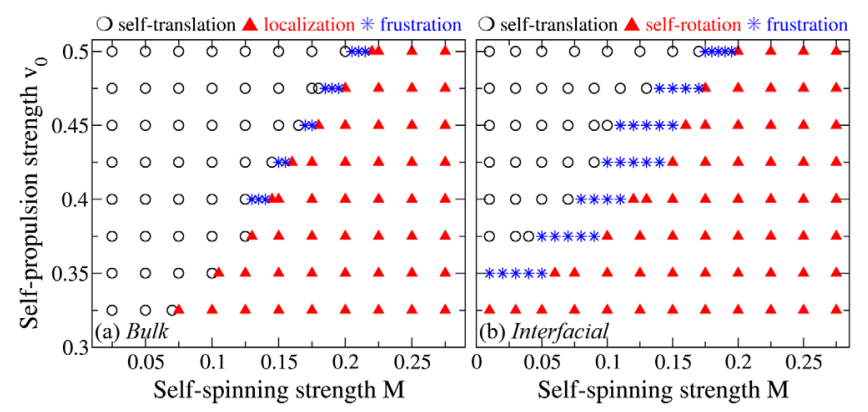

FIG. 4. Dynamical state diagrams in the $M-v_{0}$ space for three dynamical regimes in (a) bulk and (b) interfacial systems.

layers exhibit local frustration and even intermittent inverse rotation [leading to regions of local self-shearing; see Fig. 3(e) and Video S5 [49] ]. This reflects the competition among self-propulsion, self-spinning-induced localization, and interface-induced delocalization.

The dynamical regimes identified for both bulk and circularly interfacial systems are summarized in Fig. 4, in terms of $M-v_{0}$ state diagrams obtained from simulations across different values of $\psi_{0}$ that lead to hexagonal crystallized states. Major parts of the $M-v_{0}$ space are occupied by the self-translation-dominated state and the states of self-spinning-induced bulk localization [Fig. 4(a)] or interface-related crystallite self-rotation [Fig. 4(b)]. The transitional regime, characterized by dynamical frustration in both cases, is broader in Fig. 4(b) that involves crystalmelt interfaces.

The interface-induced driving mechanism of crystalline layers should apply to any geometry of nonrigid (soft) crystal-liquid boundaries or edges. In the example of a slab crystallite [Fig. 5(a)], the top and bottom interfacial layers are expected to be driven towards opposite directions due to their inverse crystal-to-liquid interface orientations, leading to self-shearing of the crystallite as verified in our simulations [see Fig. 5(a) and Video S6 [49] ]. Conversely, in the transitional regime, local frustration of density peaks occurs

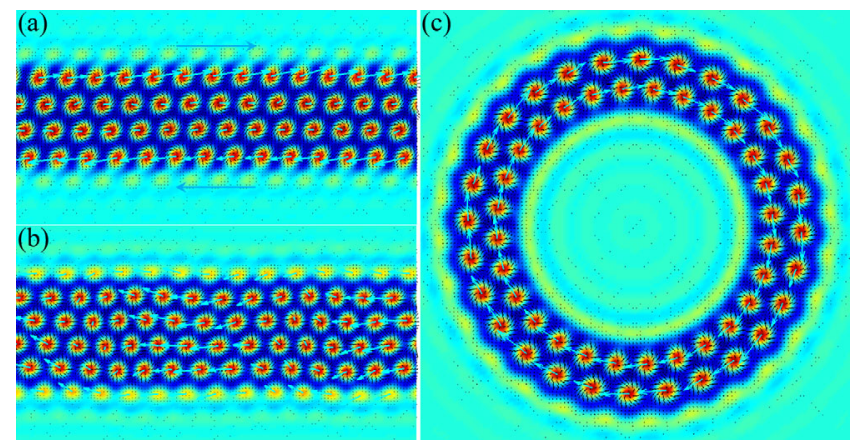

FIG. 5. Interface-induced self-motion of crystalline layers at $v_{0}=0.5$, including (a) self-shearing $\left(M=0.5, \psi_{0}=-0.2\right)$ and (b) frustrated motion $\left(M=0.19, \psi_{0}=-0.19\right)$ for a slab crystallite, and (c) self-rotation of a ring crystallite $\left(M=0.25, \psi_{0}=-0.26\right)$. 
as a consequence of the comparable strengths of selfpropulsion and self-spinning, as seen in Fig. 5(b). This leads to either fluctuating modes of traveling crystalline layers (Video S7) or even a snaking or worming type of layer flow (Video S8).

Finally, similar consequences of opposing interfacial orientations can be manifested in a ringlike configuration, as shown in Fig. 5(c) and Video S9 [49]. In many cases, although the whole ring-shaped crystallite still rotates collectively as driven by the outermost circular layer with longest perimeter [Fig. 5(c)], its angular velocity is significantly reduced due to the hindrance by the counteracting drive of the innermost annulus. The scenario of selfshearing occurs when the two interfacial drives are of comparable strength, for which the outer and inner crystalline layers rotate to opposite directions (Video S9).

In summary, we have analyzed and predicted the collective behavior of spatially ordered structures featuring both active propulsion and active rotation. The interplay between individual particle self-propulsion and self-spinning during crystallization results in various novel states of collective and persistent dynamics that are enabled by the nonrelaxational nature of the active system. The competition leads to a traveling-frustration-localization transition in active crystals with increasing strength of self-spinning, which also induces a transformation between ordered phases as a result of pattern selection. A breaking of the localization and structural arrest occurs for interfacial states, revealing persistently dynamical states of selfrotating crystallites and self-shearing or self-flowing crystalline layers. The direction of crystallite self-rotation or layer propagation is opposite to that given by the chirality of the individual self-spinning particles, an effect caused by the crystal-melt interface-induced spatial variation of local polarization and the subsequent edge-originated delocalization and collective motion of active particles.

These predictions open new possibilities to explore the emergence of novel dynamical phenomena and unveil complex mechanisms underlying a wide variety of nonequilibrium active systems governed by persistent, nonrelaxational dynamics. Although here we focused on a dry environment, the results and mechanisms identified above will in many cases still apply to leading order in additional fluid surroundings (see Supplemental Material [49]). It should even be possible to disentangle the effects of both environments in an experiment when surrounding granular or colloidal spinners by a viscous fluid with varying viscosity for the tuning of hydrodynamic couplings. Our results can be realized and verified in various experimental setups, such as a collective of granular rotors [25,28,89], light-controlled anisotropic colloidal Janus particles [90] and colloidal molecules [91], or selfpropelled particles equipped with magnetic dipole moments $[92,93]$ to perform active spinning under a magnetic field.
Z.-F.H. acknowledges support from the National Science Foundation under Grant No. DMR-1609625. A. M. M. thanks the German Research Foundation (DFG) for support through the SPP 1726, Grant No. ME 3571/2-2, and through the Heisenberg Grant No. ME 3571/4-1. H. L. was supported by the German Research Foundation (DFG) within Project No. LO 418/20-2.

[1] M. C. Cross and P. C. Hohenberg, Pattern formation outside of equilibrium, Rev. Mod. Phys. 65, 851 (1993).

[2] M. C. Cross and D. I. Meiron, Domain Coarsening in Systems far from Equilibrium, Phys. Rev. Lett. 75, 2152 (1995).

[3] Z.-F. Huang and J. Viñals, Grain boundary dynamics in stripe phases of nonpotential systems, Phys. Rev. E 75, 056202 (2007).

[4] S. W. Morris, E. Bodenschatz, D. S. Cannell, and G. Ahlers, Spiral Defect Chaos in Large Aspect Ratio Rayleigh-Bénard Convection, Phys. Rev. Lett. 71, 2026 (1993).

[5] H.-W. Xi, J. D. Gunton, and J. Viñals, Spiral Defect Chaos in a Model of Rayleigh-Bénard Convection, Phys. Rev. Lett. 71, 2030 (1993).

[6] L. F. Cugliandolo, J. Kurchan, P. Le Doussal, and L. Peliti, Glassy Behaviour in Disordered Systems with Nonrelaxational Dynamics, Phys. Rev. Lett. 78, 350 (1997).

[7] J. Schwarz-Linek, C. Valeriani, A. Cacciuto, M. E. Cates, D. Marenduzzo, A. N. Morozov, and W. C. K. Poon, Phase separation and rotor self-assembly in active particle suspensions, Proc. Natl. Acad. Sci. U.S.A. 109, 4052 (2012).

[8] B. Liebchen and D. Levis, Collective Behavior of Chiral Active Matter: Pattern Formation and Enhanced Flocking, Phys. Rev. Lett. 119, 058002 (2017).

[9] D. Levis and B. Liebchen, Simultaneous phase separation and pattern formation in chiral active mixtures, Phys. Rev. E 100, 012406 (2019).

[10] Q.-L. Lei, M. P. Ciamarra, and R. Ni, Nonequilibrium strongly hyperuniform fluids of circle active particles with large local density fluctuations, Sci. Adv. 5, eaau7423 (2019).

[11] R. Singh and M. E. Cates, Hydrodynamically Interrupted Droplet Growth in Scalar Active Matter, Phys. Rev. Lett. 123, 148005 (2019).

[12] L. Caprini, U. Marini Bettolo Marconi, and A. Puglisi, Spontaneous Velocity Alignment in Motility-Induced Phase Separation, Phys. Rev. Lett. 124, 078001 (2020).

[13] I. Buttinoni, J. Bialké, F. Kümmel, H. Löwen, C. Bechinger, and T. Speck, Dynamical Clustering and Phase Separation in Suspensions of Self-Propelled Colloidal Particles, Phys. Rev. Lett. 110, 238301 (2013).

[14] H. Karani, G. E. Pradillo, and P. M. Vlahovska, Tuning the Random Walk of Active Colloids: From Individual Run-and-Tumble to Dynamic Clustering, Phys. Rev. Lett. 123, 208002 (2019).

[15] R. Niu, A. Fischer, T. Palberg, and T. Speck, Dynamics of binary active clusters driven by ion-exchange particles, ACS Nano 12, 10932 (2018).

[16] M. N. van der Linden, L. C. Alexander, D. G. A. L. Aarts, and O. Dauchot, Interrupted Motility Induced Phase 
Separation in Aligning Active Colloids, Phys. Rev. Lett. 123, 098001 (2019).

[17] L. Berthier, Nonequilibrium Glassy Dynamics of SelfPropelled Hard Disks, Phys. Rev. Lett. 112, 220602 (2014).

[18] N. Klongvessa, F. Ginot, C. Ybert, C. Cottin-Bizonne, and M. Leocmach, Active Glass: Ergodicity Breaking Dramatically Affects Response to Self-Propulsion, Phys. Rev. Lett. 123, 248004 (2019).

[19] A. M. Menzel and H. Löwen, Traveling and Resting Crystals in Active Systems, Phys. Rev. Lett. 110, 055702 (2013).

[20] A. M. Menzel, T. Ohta, and H. Löwen, Active crystals and their stability, Phys. Rev. E 89, 022301 (2014).

[21] S. Praetorius, A. Voigt, R. Wittkowski, and H. Löwen, Active crystals on a sphere, Phys. Rev. E 97, 052615 (2018).

[22] L. Ophaus, S. V. Gurevich, and U. Thiele, Resting and traveling localized states in an active phase-field-crystal model, Phys. Rev. E 98, 022608 (2018).

[23] N. H. P. Nguyen, D. Klotsa, M. Engel, and S. C. Glotzer, Emergent Collective Phenomena in a Mixture of Hard Shapes through Active Rotation, Phys. Rev. Lett. 112, 075701 (2014).

[24] B. C. van Zuiden, J. Paulose, W. T. M. Irvine, D. Bartolo, and V. Vitelli, Spatiotemporal order and emergent edge currents in active spinner materials, Proc. Natl. Acad. Sci. U.S.A. 113, 12919 (2016).

[25] P. Liu, H. Zhu, Y. Zeng, G. Du, L. Ning, D. Wang, K. Chen, Y. Lu, N. Zheng, F. Ye, and M. Yang, Oscillating collective motion of active rotors in confinement, Proc. Natl. Acad. Sci. U.S.A. 117, 11901 (2020).

[26] K. Dasbiswas, K. K. Mandadapu, and S. Vaikuntanathan, Topological localization in out-of-equilibrium dissipative systems, Proc. Natl. Acad. Sci. U.S.A. 115, E9031 (2018).

[27] J.-C. Tsai, F. Ye, J. Rodriguez, J. P. Gollub, and T. C. Lubensky, A Chiral Granular Gas, Phys. Rev. Lett. 94, 214301 (2005).

[28] X. Yang, C. Ren, K. Cheng, and H. P. Zhang, Robust boundary flow in chiral active fluid, Phys. Rev. E 101, 022603 (2020).

[29] L. Caprini and U. Marini Bettolo Marconi, Active chiral particles under confinement: Surface currents and bulk accumulation phenomena, Soft Matter 15, 2627 (2019).

[30] A. Snezhko, Complex collective dynamics of active torquedriven colloids at interfaces, Curr. Opin. Colloid Interface Sci. 21, 65 (2016).

[31] K. Han, G. Kokot, S. Das, R. G. Winkler, G. Gompper, and A. Snezhko, Reconfigurable structure and tunable transport in synchronized active spinner materials, Sci. Adv. 6, eaaz8535 (2020).

[32] Z. Shen, A. Würger, and J. S. Lintuvuori, Hydrodynamic self-assembly of active colloids: Chiral spinners and dynamic crystals, Soft Matter 15, 1508 (2019).

[33] K. Yeo, E. Lushi, and P. M. Vlahovska, Collective Dynamics in a Binary Mixture of Hydrodynamically Coupled Microrotors, Phys. Rev. Lett. 114, 188301 (2015).

[34] W. Yang, V. R. Misko, J. Tempere, M. Kong, and F. M. Peeters, Artificial living crystals in confined environment, Phys. Rev. E 95, 062602 (2017).

[35] M. Tarama and T. Ohta, Spinning motion of a deformable self-propelled particle in two dimensions, J. Phys. Condens. Matter 24, 464129 (2012).
[36] T. Ohta, Dynamics of deformable active particles, J. Phys. Soc. Jpn. 86, 072001 (2017).

[37] C. Krüger, G. Klös, C. Bahr, and C.C. Maass, Curling Liquid Crystal Microswimmers: A Cascade of Spontaneous Symmetry Breaking, Phys. Rev. Lett. 117, 048003 (2016).

[38] A. Nourhani, S. J. Ebbens, J. G. Gibbs, and P. E. Lammert, Spiral diffusion of rotating self-propellers with stochastic perturbation, Phys. Rev. E 94, 030601(R) (2016).

[39] C. Kurzthaler and T. Franosch, Intermediate scattering function of an anisotropic Brownian circle swimmer, Soft Matter 13, 6396 (2017).

[40] O. Chepizhko and T. Franosch, Ideal circle microswimmers in crowded media, Soft Matter 15, 452 (2019).

[41] S. Fürthauer, M. Strempel, S. W. Grill, and F. Jülicher, Active Chiral Processes in Thin Films, Phys. Rev. Lett. 110, 048103 (2013).

[42] R. Kirchhoff and H. Löwen, $T$-structured fluid and jamming in driven Brownian rotators, Europhys. Lett. 69, 291 (2005).

[43] A. Kaiser and H. Löwen, Vortex arrays as emergent collective phenomena for circle swimmers, Phys. Rev. E 87, 032712 (2013).

[44] Y. Wang, S. Canic, G. Kokot, A. Snezhko, and I. S. Aranson, Quantifying hydrodynamic collective states of magnetic colloidal spinners and rollers, Phys. Rev. Fluids 4, 013701 (2019).

[45] S. Farhadi, S. Machaca, J. Aird, B. O. Torres Maldonado, S. Davis, P.E. Arratia, and D. J. Durian, Dynamics and thermodynamics of air-driven active spinners, Soft Matter 14, 5588 (2018).

[46] N. Uchida and R. Golestanian, Synchronization and Collective Dynamics in a Carpet of Microfluidic Rotors, Phys. Rev. Lett. 104, 178103 (2010).

[47] G.-J. Liao and S. H. L. Klapp, Clustering and phase separation of circle swimmers dispersed in a monolayer, Soft Matter 14, 7873 (2018).

[48] Y. Yang, F. Qiu, and G. Gompper, Self-organized vortices of circling self-propelled particles and curved active flagella, Phys. Rev. E 89, 012720 (2014).

[49] See Supplemental Material at http://link.aps.org/supplemental/ 10.1103/PhysRevLett.125.218002 for a detailed derivation of the active PFC description from a microscopic particle-based picture and DDFT, microscopic foundations of the system parameters, some remarks on the possible role of hydrodynamic interactions, and videos of various scenarios of crystallite dynamics, which includes Refs. [50-83].

[50] R. Kubo, M. Toda, and N. Hashitsume, Statistical Physics II (Springer, Berlin, 1991).

[51] H. Risken, The Fokker-Planck Equation: Methods of Solution and Applications (Springer, Berlin, 1996).

[52] R. Zwanzig, Nonequilibrium Statistical Mechanics (Oxford University Press, New York, 2001).

[53] M. Doi and S. F. Edwards, The Theory of Polymer Dynamics (Oxford University Press, New York, 1986).

[54] U. M. B. Marconi and P. Tarazona, Dynamic density functional theory of fluids, J. Chem. Phys. 110, 8032 (1999).

[55] U. M. B. Marconi and P. Tarazona, Dynamic density functional theory of fluids, J. Phys. Condens. Matter 12, A413 (2000). 
[56] A. J. Archer and R. Evans, Dynamical density functional theory and its application to spinodal decomposition, J. Chem. Phys. 121, 4246 (2004).

[57] H. H. Wensink and H. Löwen, Aggregation of self-propelled colloidal rods near confining walls, Phys. Rev. E 78, 031409 (2008).

[58] A. M. Menzel, A. Saha, C. Hoell, and H. Löwen, Dynamical density functional theory for microswimmers, J. Chem. Phys. 144, 024115 (2016).

[59] C. Hoell, H. Löwen, and A. M. Menzel, Particle-scale statistical theory for hydrodynamically induced polar ordering in microswimmer suspensions, J. Chem. Phys. 149, 144902 (2018).

[60] C. Hoell, H. Löwen, and A. M. Menzel, Multi-species dynamical density functional theory for microswimmers: Derivation, orientational ordering, trapping potentials, and shear cells, J. Chem. Phys. 151, 064902 (2019).

[61] R. Evans, Density functional theory for inhomogeneous fluids I: Simple fluids in equilibrium, in Lecture Notes $3 \mathrm{rd}$ Warsaw School of Statistical Physics, edited by B. Cichocki, M. Napiórkowski, and J. Piasecki (Warsaw University Press, Warsaw, 2010), pp. 43-85.

[62] H. Löwen, Density functional theory for inhomogeneous fluids II: Statics, dynamics, and applications, in Lecture Notes 3rd Warsaw School of Statistical Physics, edited by B. Cichocki, M. Napiórkowski, and J. Piasecki (Warsaw University Press, Warsaw, 2010), pp. 87-121.

[63] H. Löwen, A phase-field-crystal model for liquid crystals, J. Phys. Condens. Matter 22, 364105 (2010).

[64] R. Wittkowski, H. Löwen, and H. R. Brand, Derivation of a three-dimensional phase-field-crystal model for liquid crystals from density functional theory, Phys. Rev. E 82, 031708 (2010).

[65] R. Wittkowski, H. Löwen, and H. R. Brand, Polar liquid crystals in two spatial dimensions: The bridge from microscopic to macroscopic modeling, Phys. Rev. E 83, 061706 (2011).

[66] K. R. Elder and M. Grant, Modeling elastic and plastic deformations in nonequilibrium processing using phase field crystals, Phys. Rev. E 70, 051605 (2004).

[67] S. van Teeffelen, R. Backofen, A. Voigt, and H. Löwen, Derivation of the phase-field-crystal model for colloidal solidification, Phys. Rev. E 79, 051404 (2009).

[68] Z.-F. Huang, K. R. Elder, and N. Provatas, Phasefield-crystal dynamics for binary systems: Derivation from dynamical density functional theory, amplitude equation formalism, and applications to alloy heterostructures, Phys. Rev. E 82, 021605 (2010).

[69] P. F. Tupper and M. Grant, Phase field crystals as a coarsegraining in time of molecular dynamics, Europhys. Lett. 81, 40007 (2008).

[70] J. Toner and Y. Tu, Long-Range Order in a TwoDimensional Dynamical $X Y$ Model: How Birds Fly Together, Phys. Rev. Lett. 75, 4326 (1995).

[71] J. Toner and Y. Tu, Flocks, herds, and schools: A quantitative theory of flocking, Phys. Rev. E 58, 4828 (1998).

[72] R. Wittkowski, H. Löwen, and H. R. Brand, Microscopic and macroscopic theories for the dynamics of polar liquid crystals, Phys. Rev. E 84, 041708 (2011).
[73] F. Peruani, L. Schimansky-Geier, and M. Bär, Cluster dynamics and cluster size distributions in systems of selfpropelled particles, Eur. Phys. J. Special Topics 191, 173 (2010).

[74] A. M. Menzel, Collective motion of binary self-propelled particle mixtures, Phys. Rev. E 85, 021912 (2012).

[75] S. Kim and S. J. Karrila, Microhydrodynamics: Principles and Applications (Butterworth-Heinemann, Boston, 1991).

[76] J. K. G. Dhont, Introduction to Dynamics of Colloids (Elsevier, Amsterdam, 1996).

[77] D. L. Ermak and J. A. McCammon, Brownian dynamics with hydrodynamic interactions, J. Chem. Phys. 69, 1352 (1978).

[78] M. Hennes, K. Wolff, and H. Stark, Self-Induced Polar Order of Active Brownian Particles in a Harmonic Trap, Phys. Rev. Lett. 112, 238104 (2014).

[79] G. Pessot, H. Löwen, and A. M. Menzel, Binary pusherpuller mixtures of active microswimmers and their collective behaviour, Mol. Phys. 116, 3401 (2018).

[80] T. Bäuerle, A. Fischer, T. Speck, and C. Bechinger, Selforganization of active particles by quorum sensing rules, Nat. Commun. 9, 3232 (2018).

[81] F. A. Lavergne, H. Wendehenne, T. Bäuerle, and C. Bechinger, Group formation and cohesion of active particles with visual perception-dependent motility, Science 364, 70 (2019).

[82] R. D. L. Hanes, M. C. Jenkins, and S. U. Egelhaaf, Combined holographic-mechanical optical tweezers: Construction, optimization, and calibration, Rev. Sci. Instrum. 80, 083703 (2009).

[83] I. Williams, E. C. Oğuz, T. Speck, P. Bartlett, H. Löwen, and C.P. Royall, Transmission of torque at the nanoscale, Nat. Phys. 12, 98 (2016).

[84] K. R. Elder, M. Katakowski, M. Haataja, and M. Grant, Modeling Elasticity in Crystal Growth, Phys. Rev. Lett. 88, 245701 (2002).

[85] K. R. Elder, N. Provatas, J. Berry, P. Stefanovic, and M. Grant, Phase field crystal modeling and classical density functional theory of freezing, Phys. Rev. B 75, 064107 (2007).

[86] S. K. Mkhonta, K. R. Elder, and Z.-F. Huang, Exploring the Complex World of Two-Dimensional Ordering with Three Modes, Phys. Rev. Lett. 111, 035501 (2013).

[87] S. K. Mkhonta, K. R. Elder, and Z.-F. Huang, Emergence of Chirality from Isotropic Interactions of Three Length Scales, Phys. Rev. Lett. 116, 205502 (2016).

[88] C. Hoell, H. Löwen, and A. M. Menzel, Dynamical density functional theory for circle swimmers, New J. Phys. 19, 125004 (2017).

[89] C. Scholz, M. Engel, and T. Pöschel, Rotating robots move collectively and self-organize, Nat. Commun. 9, 931 (2018).

[90] F. Kümmel, B. ten Hagen, R. Wittkowski, I. Buttinoni, R. Eichhorn, G. Volpe, H. Löwen, and C. Bechinger, Circular Motion of Asymmetric Self-Propelling Particles, Phys. Rev. Lett. 110, 198302 (2013).

[91] F. Schmidt, B. Liebchen, H. Löwen, and G. Volpe, Lightcontrolled assembly of active colloidal molecules, J. Chem. Phys. 150, 094905 (2019). 
[92] L. Baraban, D. Makarov, R. Streubel, I. Monch, D. Grimm, S. Sanchez, and O. G. Schmidt, Catalytic Janus motors on microfluidic chip: Deterministic motion for targeted cargo delivery, ACS Nano 6, 3383 (2012).
[93] R. Taukulis and A. Cebers, Diffusion in active magnetic colloids, J. Magn. Magn. Mater. 368, 428 (2014). 